\title{
Humeur de chaos
}

Mme Denise Pumain

Citer ce document / Cite this document :

Pumain Denise. Humeur de chaos. In: Espace géographique, tome 19-20, n4, 1990. pp. 309-310;

doi : $10.3406 /$ spgeo.1990.3009

http://www.persee.fr/doc/spgeo_0046-2497_1990_num_19_4_3009

Document généré le 06/09/2016 


\title{
Humeur de chaos
}

\author{
Denise Pumain, Université de Paris 1
}

Je ne cacherai pas mon amusement devant l'importance que prennent les effets de mode dans le discours scientifique. Parce que chaos dans le langage courant veut dire aussi désordre, parce que pour certains qui l'attendent ou le redoutent peut-être le désordre signifie l'anarchie, nous voilà contraints à nous pencher sur ces êtres mathématiques étranges que sont les processus chaotiques, au comportement imprévisible quoique issu de modèles déterministes.

On ne me soupçonnera pas, je l'espère, de jouer les esprits chagrins qui répugnent à la ludique mathématique, ou conservateurs qui refusent d'assimiler la nouveauté... À tout hasard, je rappellerai plusieurs écrits (Pumain, Saint-Julien, Sanders, 1987; Pumain, 1989) où, comme tant d'autres, nous attirions l'attention sur l'intérêt pour la géographie de la théorie des bifurcations ou de la synergétique... Il y a à cela des raisons théoriques, pour ne pas dire paradigmatiques, puisque la physique d'aujourd'hui redécouvre, au centre de la conceptualisation, les idées d'irréversibilité des trajectoires, d'unicité des histoires, d'imprévisibilité des devenirs locaux et d'interdépendance entre les niveaux d'échelle. Mais ces idées-là, à propos des systèmes humains, sont presque aussi vieilles que la pensée! Ce qui est nouveau, et intéressant pour nous, c'est la possibilité de les faire passer à l'épreuve des faits, pour en apprendre davantage sur la dynamique des objets géographiques, au moyen de modèles mathématiques désormais capables de transcrire ces idées en équations et en paramètres.

Méfions-nous des présentations théoriques autojustificatives. La théorie mathématique est utile aux mathématiciens. Il est de notre responsabilité de découvrir, parmi toutes les propositions théoriques énoncées par les autres sciences, quelles sont celles qui ont quelque chance de nous aider à préciser et améliorer l'état de nos propres théories géographiques. Selon D. Ruelle (1991), l'un des «inventeurs» du chaos (Ruelle, Takens, 1971), «ce que l'on appelle maintenant chaos est une évolution temporelle avec dépendance sensitive des conditions initiales... dans beaucoup de cas (écologie, économie, sciences sociales), même si l'on arrivait à écrire des équations d'évolution temporelle, ces équations devraient changer lentement avec le temps parce que le système apprend et change de nature. Pour de tels systèmes, l'impact du chaos reste au niveau de la philosophie scientifique plutôt qu'au niveau de la science quantitative» (p. 89 et 105).

Autrement dit, quel est le champ d'application possible en géographie de la théorie mathématique du chaos? C'est la première condition à vérifier avant de procéder à un transfert des concepts associés et de les adapter à des applications. Selon D. Ruelle, «ce sont les évolutions temporelles avec "éternel retour" qui constituent le domaine naturel d'application des idées du chaos. Pour ces évolutions, le système revient inlassablement près des mêmes situations» (p. 115). Avons-nous à nous préoccuper des oscillations d'un pendule? ou de la trajectoire des astres? Tout au plus pouvons-nous imaginer d'appliquer les idées du chaos aux dynamiques relatives des entités géographiques, après "soustraction» de ce qui fait leur devenir historique (Dendrinos, Sonis, 1990), et qui sait si, ce faisant, nous ne passons pas à côté de l'essentiel?

Plus pertinente encore est la question de savoir si les exemples de comportement chaotique que l'on nous présente à propos de structures spatiales ont quelque chance d'intervenir dans le champ normal de variation de leurs paramètres. Quelle est l'utilité d'un modèle qui prédit l'émergence d'un comportement chaotique d'un système de migrations interrégionales au bout de mille ans? (Rogerson, 1985, Courgeau, 1990). Ou d'un modèle de prévision de la distribution du commerce de détail dans une ville qui suppose possible une vitesse de substitution 
de l'ordre de la journée, voire du quart d'heure? (White, 1985). Ou encore, d'un modèle de croissance logistique ou proie-prédateur qui devient chaotique si l'un des taux de croissance dépasse 350\% (May, 1976)? - ce qu'A. Dauphiné a le mérite de rappeler. Tous les auteurs qui ont testé un modèle susceptible de produire un comportement chaotique, le plus souvent inspiré de l'équation de May (1976), dans une perspective d'application à des questions d'ordre géographique, régional (Nijkamp, Poot, 1987; Dendrinos et Sonis, 1987) ou urbain (Dendrinos et Mullaly, 1985) ont souligné la très faible probabilité d'observer dans la réalité les valeurs des paramètres nécessaires pour l'apparition du chaos. En microéconomie, peut-être, des applications seraient possibles (Day, 1981; Baumol et Benhabib, 1989).

Je suivrai donc F. Durand-Dastès quand il invite à s'interroger sur cette absence: si les équations qui décrivent par ailleurs convenablement la dynamique d'un système sont susceptibles, dans un autre contexte, d'engendrer un comportement chaotique, qu'est-ce qui empêche l'apparition du chaos dans les conditions ordinaires? Comment les systèmes s'arrangent-ils pour se comporter de manière régulière et sans à-coups en dépit de leurs non-linéarités intrinsèques? Quels sont les mécanismes qui régissent la stabilité? Selon D. Ruelle (1991) «l'histoire ne se répète pas. Pour les systèmes très complexes et sans récurrence, on a généralement dépendance sensitive des conditions initiales mais le problème se pose de savoir si celle-ci est limitée par des mécanismes régulateurs, ou si elle donne lieu à des effets importants à long terme» (p. 110).

Peut-être y aura-t-il quelque utilité à suivre les travaux des informaticiens, qui se penchent sur la gestion des très grands systèmes, et qui cherchent précisément les moyens d'éviter les dérives chaotiques dues aux erreurs et aux retards dans la transmission des informations. Leurs premiers résultats sont intuitivement encourageants (Huberman, 1990): dans les systèmes qui ont un grand nombre de degrés de liberté, on empêche le chaos en préservant une certaine diversité parmi les agents du système, et en introduisant un mécanisme qui récompense les stratégies efficaces, de façon à ce que leur nombre augmente aux dépens des autres: cela ne vous rappelle-t-il rien?

\section{Références}

BAUMOL W.J., BENHABIB J. (1989). «Chaos: significance, mechanism, and economic applications». Journal of Economic Perspectives, $\mathrm{n}^{\circ} 3$, p. 77-105.

COURGEAu D. (1990). Un modèle d'analyse et de prévision de l'évolution des migrations interurbaines. Actes du colloque de Rabat. Paris: INED-AIDELF.

DAY R.H. (1981). "Emergence of chaos from classical economic growth". Geographical Analysis, vol. 13, n 4, p. 315-327.

Day R.H., DasGuPTA S., DutTa S.K., Nugent J.B. (1987). "Instability in rural-urban migration". The Economic Journal, n' 97.

DENDRINOS D.S., Mullaly H. (1985). Urban Evolution: Studies in the Mathematical Ecology of Cities. Oxford: Oxford University Press.

DENDRINos D.S., Sonis M., (1987). "The Onset of Turbulence in Discrete Relative Multiple Spatial Dynamics." Applied Mathematics and Computation, $\mathrm{n}^{\circ} 22$, p. 25-44.

DendRINos D.S., Sonis M. (1990). Chaos and Socio-Spatial Dynamics. New-York: Springer Verlag.

HuBERMAN R. (1990). "The performance of cooperative processes." Physica, D 42, p. 38-47.

LORENZ E. (1963). "Deterministic non-periodic flows". Journal Atmos. Sci., n 20, p. 130.

MAY R.M. (1976). "Simple mathematical model with very complicated dynamics". Nature, $n^{\circ} 261$, p. 459-467.

NuJkamp P., PоOт J. (1987). "Dynamics of generalized spatial interaction models". Regional Science and Urban Economics, $\mathrm{n}^{\circ}$ 17, p. 367-390.

Pumain D., Saint-Julien T., Sanders L. (1987). "Applications of a dynamic urban model". Geographical Analysis, vol. 19, p. 152-166.

Pumain D. (1989). "Spatial dynamics and urban models". In Hauer J. Timmermans H. Wrigley N. eds. Urban Dynamics and Spatial Choice Behaviour. Dordrecht: Kluwer, p. 155-173.

Rogerson P.A. (1985). "Disequilibrium adjustment processes and chaotic dynamics". Geographical Analysis, vol. 17, p. 185-198.

ROSSER J.B. (1990). "Approaches to the Analysis of the Morphogenesis of Regional Systems". Socio-spatial Dynamics, vol. 1, $\mathrm{n}^{\circ}$ 2, p. 75-102.

ROSSER J.B. (1991). From Catastrophe Theory to Chaos: A general Theory of Economic Discontinuities. New York: Kluwer.

Ruelle D. (1991). Hasard et chaos. Paris: Odile Jacob.

Ruelle D., TAKens F. (1971). "On the nature of turbulence". Communications in Mathematical Physics, $\mathrm{n}^{\circ} 20$, p. 167.

WHITE R. W. (1985). "Transitions to chaos with increasing system complexity: the case of regional industrial systems". Environment and Planning A, $\mathrm{n}^{\circ}$ 17, p. 387-396. 